\title{
Learning-Based Trajectory Optimization for 5G mmWave Uplink UAVs
}

\author{
Praneeth Susarla*, Yansha Deng ${ }^{\dagger}$, Giuseppe Destino*†, Jani Saloranta*, \\ Toktam Mahmoodi ${ }^{\dagger}$, Markku Juntti*, Olli Sílven* \\ *University of Oulu, Finland \\ $\dagger$ King's College London, United Kingdom \\ email: praneeth.susarla@oulu.fi
}

\begin{abstract}
A Connectivity-constrained based path planning for unmanned aerial vehicles (UAVs) is proposed within the coverage area of a 5G NR Base Station (BS) that uses mmWave technology. We consider an uplink communication between UAV and BS under multipath channel conditions for this problem. The objective is to guide a UAV, starting from a random location and reaching its destination within the BS coverage area, by learning a trajectory alongside achieving better connectivity. We propose simultaneous learning-based path planning of UAV and beam tracking at the BS side under urban macro-cellular (UMa) pathloss conditions, to reduce its sweeping time with apriori computational overhead using the deep reinforcement learning method such as Deep Q-Network (DQN). Our results show that our proposed learning-based joint path planning and beam tracking method is on par with the learning-based shortest path planning, besides beam tracking comparable to heuristic exhaustive beam searching method.
\end{abstract}

Index Terms-5G, mmWave, reinforcement learning, UAVs, path planning, beamforming

\section{INTRODUCTION}

Over the last few years, there is tremendous research interests towards integrating unmanned aerial vehicles (UAVs) into cellular networks using fifth Generation (5G) and beyond wireless communications [1], [2]. The deployment of cellularenabled UAV-User Equipment (UE)s (here after addressed as $U A V s$ ) adds an additional dimension of challenges in the design and planning of these communication systems for high altitude coverages in a myriad of civil applications, such as traffic surveillance, mineral exploration, internet drone delivery systems, etc. [3], [4]. For instance, Amazon is projected to deploy a commercial fleet of 450,000 drones under "Amazon Prime Air" worldwide delivery operating service by 2020 [5].

Millimeter wave (mmWave) beamforming communication is considered as one of the key innovations for $5 \mathrm{G}$ networks [6], [7]. The wide spectrum (ranging from $30 \mathrm{GHz}$ to 300 $\mathrm{GHz}$ ) of mmWave frequencies with beamforming has provided a promising way for sustaining a real-time ultra-high speed transmissions for UAVs. Besides, mmWave provides highcapacity and line-of-sight (LoS) dominated connectivity for UAVs, it also suffers from many challenges, such as rapid channel variation, blockage effects, atmospheric attenuations, range limits etc. [8], [9], leading to substantial interference problems especially in multi-cell scenarios. The recent studies have even reported nearly $150 \mathrm{UAV}$ crashes occurred due to loss of communication with ground base stations [10].
As there is an expected increase of UAVs in near future, it is of significant importance to support high-performance communications between UAVs and Base Station (BS).

Connectivity-constrained trajectory optimization for UAVmounted relays and UAV-mounted base stations in cellular networks has been widely investigated in [11]-[14]. In [11], a minimal delay trajectory design for UAV relays was proposed to ferry data from multiple sources to destination using reinforcement learning (RL) algorithm. In [13], a multiUAV enabled multi user communication system was jointly optimized with UAV trajectory and communication resource allocation to maximize throughput over all users for downlink transmission scenario. The authors in [14], proposed a deep RL framework based on echo state network (ESN) cells for optimizing the trajectories in a multi-cellular UAV scenario. This approach allowed UAVs act as individual RL agents to minimize their interference on ground network under latency.

In this paper, we consider a connectivity-constrained based trajectory optimization for any cellular-enabled UAV within the coverage area of mmWave BS, using deep RL framework. Unlike in previous works, here the mmWave BS acts as RL agent to learn communication-aware optimal trajectory and optimal beam tracking for UAV. Some literature has recently studied the use of RL for mmWave beam learning in an online manner. [15], [16]. The deep RL framework offers a generic and scalable solution, where BS acts as a commander for all UAVs in a multi-UAV scenario. Besides, the smart BS approach for cellular-enabled UAVs can comply well with current UAV battery standards [17].

The main contribution of this paper is that we propose a deep RL based generic framework at BS side, to jointly optimize path planning and beam tracking for any uplink mmWave cellular-enabled UAV. This communication is simulated by considering a multi-path channel model, Multiple Inputs Multiple Outputs (MIMO) beamformers and UAV-BS environment. The simulated environment is used for offline training of BS RL agent before deployment similar to prior works [11]-[14]. The trained agent is used to benchmark our results against learning-based shortest path planning with heuristic exhaustive beam searching method.

The rest of the paper is organized as follows. Section II presents the system and communication model with the problem formulation. Section III describes the implementation of 
our deep RL framework and UAV environment. Section IV elaborates our simulations and benchmark results. Section V summarizes our conclusion and future work.

\section{Problem Formulation and System Model}

As illustrated in Fig 1, we consider a mmWave uplink multi-path (both LoS and Non-Line of Sight (NLoS)) radio beamforming communication between a fixed BS and a moving unmanned aerial vehicle (UAV), following $5 \mathrm{G}$ protocol standards as mentioned in [18]. UAV starts from a random source location with the goal to reach destination by assuming all the locations hovered by UAV (as UE) are within the coverage area of BS. The objective of this problem is to guide the UAV via BS in reaching destination, by predicting the next best UE location as well as BS radio frequency (RF) beam direction based on its connectivity-constraints.

\section{A. System Model}

We consider a multi-antenna UE, multi-antenna BS scenario and also conceptualize the coverage area $\mathbb{U}$ around BS into a grid as shown in Fig 1(a). The BS acts as fixed serving node located at $\mathcal{O}(0,0)$. UE transmits a radio signal in multiple beam directions following a codebook $\mathcal{B}$ defined as

$$
b_{i}=(i-1) \frac{\pi}{N-1}, 1 \leq i \leq N,
$$

where $b_{i}$ represents a $\mathrm{RF}$ radio beam direction with a fixed narrow beam width $\left(\frac{\pi}{N-1}\right), N$ represents the number of codebook directions in $\mathcal{B}$. BS receives the radio signal through one of its multiple beam directions each time, following the same codebook set $\mathcal{B}$. UE starts from source location $\mathrm{UE}_{s}$ and moves with velocity $v=\left(v_{x}, v_{y}, v_{z}\right)\left(\left|v_{x}\right|,\left|v_{y}\right|,\left|v_{z}\right| \in \mathcal{V}\right.$, where $\mathcal{V}$ denote the set of UE pre-defined speed values) towards a defined target location $\mathrm{UE}_{d}$, following a certain path $p$. Meanwhile, the RX radio unit starts with a random beam $b_{r} \in \mathcal{B}$ at time $t=0$ and learns to choose the beam direction $b_{k} \in \mathcal{B}$ every time, until reaching the target location at along the path $p$. Let $\mathcal{M} \in\left\{{ }^{\prime} L{ }^{\prime},{ }^{\prime} R\right.$ ' ' $U$ ', ' $D$ ' $\}$ represents the set of possible UE move directions under the coverage area $\mathbb{U}$. If $\mathrm{UE}_{s}$ is denoted as $\left(x_{1}, y_{1}, z_{1}\right)$, then the position of UE via $\mathrm{BS}$ at any time instant $t$ is given by

$$
\mathrm{UE}_{t}=\left(x_{1}+v_{x} t, y_{1}+v_{y} t, z_{1}+v_{z} t\right), \forall 1 \leq t \leq t_{p},
$$

where $\left\{x_{1}+v_{x} t, y_{1}+v_{y} t\right\} \in \mathbb{U}$ and $\left\{z_{1}+v_{z} t\right\} \in \mathbb{Z}$, depending on UE move direction and $t_{p}$ is the maximum time step limit. Here, $\mathbb{U}$ and $\mathbb{Z}$ is the coverage area of the the serving Node BS and altitude range of UAV respectively.

\section{B. Communication Model}

We consider an uplink MIMO mmWave communication between $\mathrm{UE}$ at location $\mathrm{UE}_{t} \in \mathbb{R}^{2}$ and $\mathrm{BS}$ at location $\mathcal{O} \in \mathbb{R}^{2}$, as shown in Fig. 1(b). UE acts as transmitter (TX), BS as receiver (RX) and are equipped with Uniform Linear Array (ULA) structure of $N_{t}$ and $N_{r}$ antennas respectively. We assume $\mathrm{UE}_{t}$ is unknown and estimated using (2) while BS location is known as $\mathcal{O}(0,0)$. The radio channel considered in this problem is a multi-ray link ( $\operatorname{LoS}$ and NLoS) with

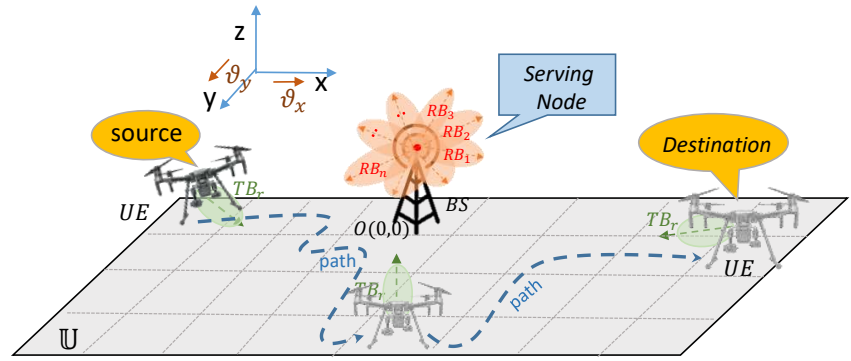

(a)

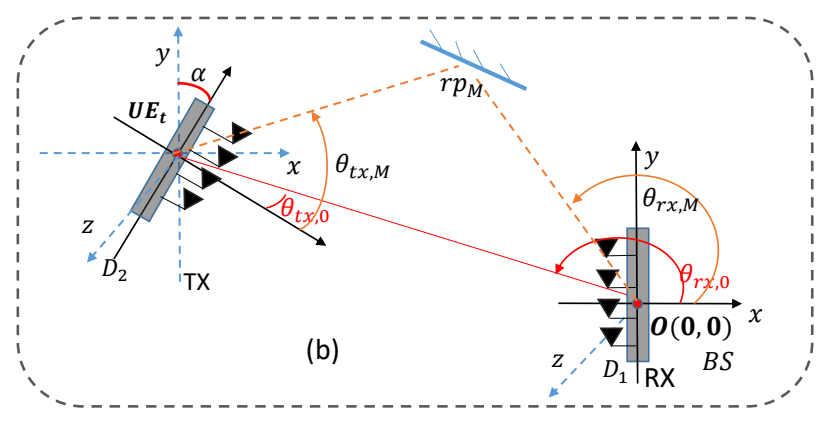

Fig. 1. (a) Illustration of problem formulation, (b) Radio Communication system between MIMO transmitter and receiver.

propagation delay $\tau_{m} \forall 0 \leq m \leq M$, where $M$ represents the number of reflection points between UE and BS. Let $\alpha \in[0,2 \pi)$ be the angle of rotation of UE antenna array with respect to y-axis. $\theta_{\mathrm{tx}, \mathrm{m}}, \theta_{\mathrm{rx}, \mathrm{m}}$ are the Angle of Departure (AoD) and Angle of Arrival (AoA) of $m^{\text {th }}$ communication link between BS and UE respectively. Using these notations, we can now define $\alpha=\pi+\theta_{\mathrm{tx}, \mathrm{m}}-\theta_{\mathrm{rx}, \mathrm{m}}$ and propagation delay $\tau_{m}$ with velocity of light $c$ is given by $\tau_{m}=\frac{\left\|\mathrm{UE}_{t, m}\right\|}{c}$.

UE transmits radio signals in all codebook directions at a carrier frequency $f_{c}$ (or wavelength $\lambda$ ) with bandwidth $W$. The BS receives carrier $f_{c}$ signal through one of its multiple beams defined under same direction set $\mathcal{B}$ using (1). Based on this, we employ a narrow band receiver signal model given by

$$
\begin{array}{r}
y(t)=\sum_{m=0}^{M} \sqrt{P_{t x}} \beta \mathbf{w}^{H} \mathbf{a}_{R}\left(\theta_{\mathrm{rx}, \mathrm{m}}\right) \mathbf{a}_{T}^{H}\left(\theta_{\mathrm{tx}, \mathrm{m}}\right) \mathbf{f} x\left(t-\tau_{m}\right) \\
+\mathbf{w}^{H} n(t),
\end{array}
$$

where $P_{t x}$ is transmission power, $\beta$ is the antenna channel gain with UMa environment multi-path loss conditions [19], w $\in \mathbb{C}^{N_{r}}, \mathbf{f} \in \mathbb{C}^{N_{t}}$ are the transmit and receive unit-norm beamforming vectors, $n(t) \in \mathcal{C}^{N_{r}}$ is a Gaussian noise vector with zero mean and two-sided power spectral density $\frac{N_{0}}{2}, x(t)$ represents one Orthogonal Frequency Division Multiple access (OFDM) symbol of the timedomain transmitted signal with bandwidth $W$ and time period $T_{\mathrm{sym}}$ with $\frac{1}{T_{\mathrm{sym}}} \int_{0}^{T_{\mathrm{sym}}}\|x(t)\|^{2} d t=1, \mathbf{a}_{R}\left(\theta_{\mathrm{rx}, \mathrm{m}}\right) \in \mathbb{C}^{N_{r}}$, $\mathbf{a}_{T}\left(\theta_{\mathrm{tx}, \mathrm{m}}\right) \in \mathbb{C}^{N_{t}}$ are the array response vectors for $\theta_{\mathrm{rx}, \mathrm{m}}$ and $\theta_{\mathrm{tx}, \mathrm{m}}$ along $m^{\text {th }}$ communication path. Here, $\left.\mathbf{a}(\theta)\right]_{l=0}^{N-1}=$ $\frac{1}{\sqrt{N}} \exp \left(j \frac{2 \pi l d}{\lambda} \sin (\theta)\right.$, where $\theta=\theta_{\mathrm{rx}, \mathrm{m}}, N=N_{r}$ and $\theta=$ 
$\theta_{\mathrm{tx}, \mathrm{m}}, N=N_{t}$ for $\mathbf{a}_{R}\left(\theta_{\mathrm{rx}, \mathrm{m}}\right)$ and $\mathbf{a}_{T}\left(\theta_{\mathrm{tx}, \mathrm{m}}\right)$, respectively. If $N_{\text {FFT }}$ represents the number of OFDM subcarriers, then signal-to-noise ratio (SNR) can be defined as

$$
\mathrm{SNR}=\sum_{n=0}^{N_{\mathrm{FFT}}} \sum_{m=0}^{M} \frac{|\beta|_{n}^{2} P_{\mathrm{tx}}\left|\mathbf{w}^{H} \mathbf{a}_{R}\left(\theta_{\mathrm{rx}, \mathrm{m}}\right)\right|_{n}^{2}\left|\mathbf{f}^{H} \mathbf{a}_{T}\left(\theta_{\mathrm{tx}, \mathrm{m}}\right)\right|_{n}^{2}}{N_{0} W}
$$

and overall rate measurement $R$ is given by

$$
R=W \log (1+\mathrm{SNR}) .
$$

\section{Problem Formulation}

We now formulate the problem to guide UE along a path via BS, with the goal to reach its destination using its mobility and data rate measurements (4). At any time instant $t$, we define the parameters, $o_{t}=\left\{a_{t-1}, R_{t-1}, a_{t-2}, R_{t-2}, \ldots, a_{1}, R_{1}\right\}$ that denote observation history of previous data rate measurements $R_{t}$ and $a_{t}$ are the action parameters $a_{t}=\left(\eta_{t}, b_{t}\right)$ where $\eta_{t} \in \mathcal{M}$ represents UE move direction and $b_{t} \in B$ represent receiver beam direction from (1).

The BS aims at maximizing the long-term average of both connectivity and path guidance to UE by following a stocastic policy $\pi$, mapping current observation history $o_{t}$ to selected action parameters probabilities $a_{t}$. The goal of BS is to serve UAV with an optimal path guidance as well as provide better connectivity at every instant along the path. We consider the connectivity and path constraints in terms of data rate measurements between UAV-BS and distance measurements with respect to destination location respectively. Therefore, in order to achieve the desired goal, we need to find an optimal policy that can maximize the data rate measurements by moving closer towards destination at every instant along the path. Based on this, optimization problem can now be formulated as

$$
(P 1): \max _{\left\{\pi\left(a_{t} \mid o_{t}\right)\right\}} \sum_{k=t}^{\infty} \gamma^{k-t} \mathbb{E}_{\pi}\left[\frac{R_{t}}{\delta_{t}}\right],
$$

where $\gamma \in[0,1)$ is the discount factor for future constraints along the path reaching destination, $R_{t}$ corresponds to the data rate measurements of $\mathrm{UE}_{t}$ while $\delta_{t}$ is the $\mathrm{UE}_{t}$ distance from destination. Here, the expectation operator $\mathbb{E}$ helps in providing the long-term average both connectivity and path constraint measurements as $t \rightarrow \infty$. Since, the dynamics of the system is Markovian over time and is defined by DQN flowchart to be further discussed below as shown in Fig. 2, this is considered as a Partially Observable Markov Decision Process (POMDP) problem [20] that is generally intractable. Approximate solution using deep RL method will be discussed in Section. III.

\section{IMPLEMENTATION}

As shown in Fig. 2, we apply a DQN [21] learning framework for the uplink radio communication with BS as a learning Q-agent and UE as the environment. The objective is to predict the immediate UE direction along with its RX beam direction using its distance from target and current data rate measurement information.
We consider $s_{t} \in \mathcal{S}, o_{t} \in \mathcal{O}, a_{t} \in \mathcal{A}$ and $r_{t} \in \mathcal{R}$ as any state, observation, action and reward at time instant $t$, from their corresponding sets, respectively. The BS-agent observes the current state $s_{t}$ corresponding to the observations history $o_{t}$ and selects a specific action $a_{t} \in \mathcal{A}\left(s_{t}\right)$ following standard DQN procedure. Here, $s_{t}$ represents set of indices mapping to $\mathrm{UE}_{t-1}$ location and $a_{t}$ corresponds to $\left(\eta_{t}, b_{t}\right)$ pair described under Section II-C. Once an action $a_{t}$ is performed on the environment, the Q-agent will receive a scalar reward $r_{t+1}$ observing a new state $s_{t+1}$. As our goal is to optimize the UE path guidance, the reward function should be defined according to the considered constraints such data rate measurements $R_{t}$ and distance from destination $\delta_{t}$ as

$$
r(t+1)=\log _{10}\left(\frac{R_{t}}{\delta_{t}}\right) .
$$

In this section, we introduce the Neural Networks (NN) architecture, used as the DQN-Agent at BS and learn the desired goal using the optimization function described in $P 1$. At every iteration, the UAV environment computes the subsequent states and rewards (6) for DQN-Agent based on the UE target distance and data rate measurements (4).

\section{A. UAV Environment}

We implement a UAV custom environment (denoted as UAVEnv) using python framework and OpenAI gym interface [22]. UAVEnv consists of defined coverage area $\mathbb{U}$ of the BS using 2D discrete state space $S$ (2), radio beam direction set $\mathcal{B}(1)$, possible UE directions $\mathcal{M}$ and discrete action space $\mathcal{A}$. The $\mathrm{BS}$ and target location $\left(\mathrm{UE}_{d}\right)$, multi-path channel model $h$ with UMa conditions, received signal (3) and data rate measurements (4) are also implemented under UAVEnv class interface. At each episode, UAVEnv resets to random source location $\mathrm{UE}_{s}$, computes new observations and rewards based on the received action until it reaches destination.

\section{B. $D Q N$ Architecture}

DQN is a value-based RL approach [21], learning an optimal approximated policy of states mapping to actions $\pi(s)=a$ by parameterizing and estimating state-action value function $Q(s, a ; \theta)$ using Deep Neural Networks (DNN). We denote the primary DNN network weight matrix and target DNN network weight matrix as $\theta$ and $\bar{\theta}$, respectively [21]. We consider a fully connected DNN for both the networks where $\bar{\theta}$ is updated with primary network parameters $\theta$, after every $K$ iterations. The input of DNN is given by the variables in $s_{t}$. The intermmediate layers are fully connected linear units with Rectifier Linear Units (ReLU) by using the function $f(x)=\max (0, x)$ while the output layer is composed of linear units, which are in one-one corresponding relationship with all available actions in $\mathcal{A}$. We consider initialization of bias and weights of these layers with zeros and Kaiming normalization [23], respectively.

At a time instant $t, a_{t}$ selects either a random action from $\mathcal{A}$ or perform forward propagation of $Q\left(s_{t}, a_{t} ; \theta\right)$ following $\epsilon$-greedy policy [20]. A memory buffer of experiences $D_{t}=$ 


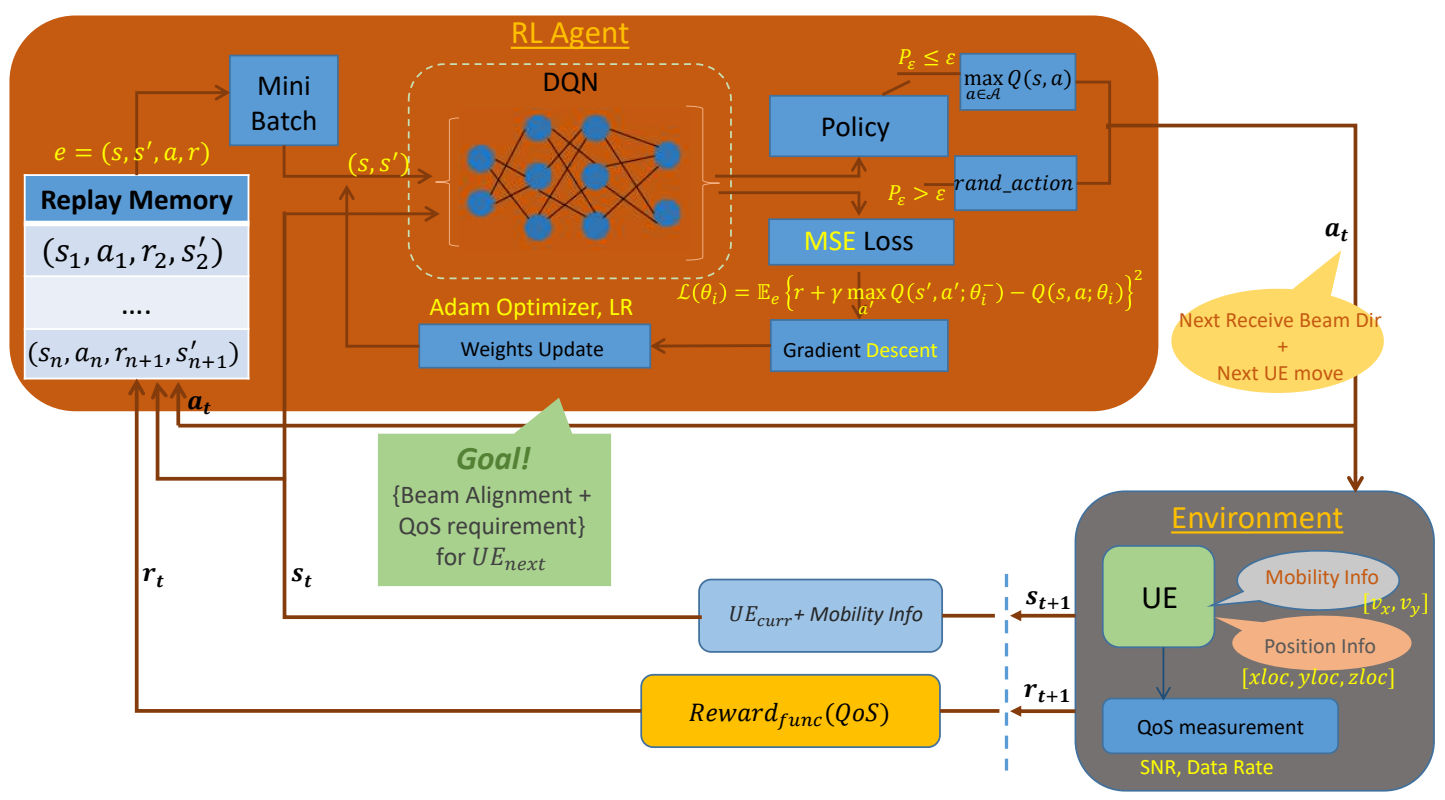

Fig. 2. Flowchart of the DQN learning scenario.

$\left\{e_{1}, e_{2}, e_{3}, \ldots, e_{t}\right\}, e_{i}=\left(s_{i}, a_{i}, r_{i+1}, s_{i+1}\right)$ are collected where a mini batch of them $U(D)$ are randomly sampled and sent into DQN [21]. During back propagation, a Mean Squared Error (MSE) loss function is computed between primary, target networks and $\theta$ is updated using Stocastic Gradient Descent (SGD) [24] and Adam Optimizer [25] as

$$
\theta_{t+1}=\theta_{t}-\xi_{\mathrm{Adam}} \nabla \mathcal{L}^{\mathrm{DQN}}\left(\theta_{t}\right),
$$

where $\xi_{\text {Adam }}$ is the learning rate. $\nabla \mathcal{L}\left(\theta_{t}\right)$ is the gradient of the DQN loss function, given as

$$
\begin{aligned}
& \nabla \mathcal{L}^{\mathrm{DQN}}\left(\theta_{t}\right)= \\
& \underset{\left(s_{i}, a_{i}, r_{i+1}, s_{i+1}\right)}{\mathbb{E}}\left[\left(R_{i+1}+\gamma \max _{a} Q\left(s_{i+1}, a ; \bar{\theta}_{t}\right)\right.\right. \\
&\left.\left.-Q\left(s_{i}, a_{i} ; \theta_{t}\right)\right) \nabla_{\theta} Q\left(s_{i}, a_{i} ; \theta_{t}\right)\right],
\end{aligned}
$$

where $\bar{\theta}_{t}$ is used to estimate future value of Q-function inside $\mathcal{L}^{\mathrm{DQN}}$. Complete steps followed by DQN for every episode of our path planning problem is shown in Algorithm. 1.

\section{Simulation Results}

In this section, we evaluate the performance of the proposed DQN algorithm for our path planning problem. We compare our learning-based joint path planning and beam tracking method against learning-based path planning with heuristic exhaustive beam searching method [26]. We adopt standard radio channel modelling parameters for mmWave communications as well as grid environment parameters listed together in Table I. The hyper-parameters used for DQN learning algorithm are also listed in Table II.

As our state space dimension is smaller $\left(\mathrm{UE}_{x}, \mathrm{UE}_{y}\right)$, for now we consider a three layer network in our implementation. However, with increase in action space $\mathcal{A}$ and state space $S$ for more generalization, more layers in DNN's have to be

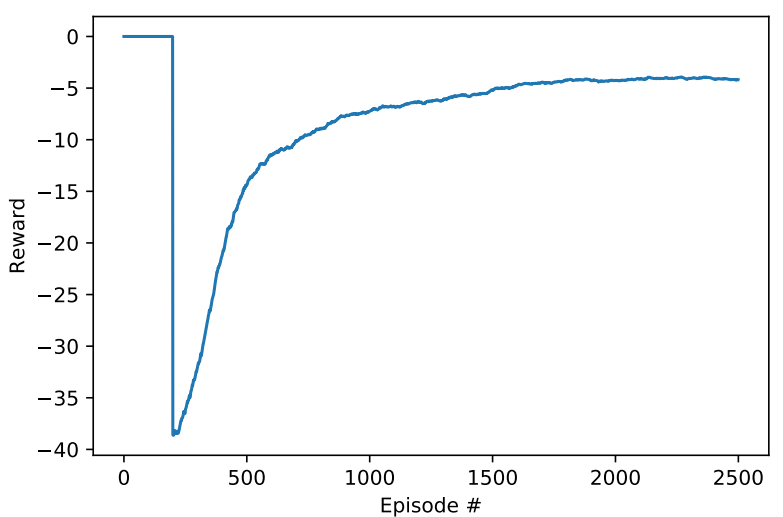

Fig. 3. Average received rewards against DQN training episodes for BS agent

used. Thus, our DQN architecture is set with two 128 hidden layers and one 64 hidden layer ReLU units, respectively. Also, we assume a $2 D$ uplink beamforming communication between UAV and BS by considering the altitude of UAV fixed. Fig. 3 plots the received episodic reward over training episodes, smoothened for every 200 samples (given as $\mathbb{E}\{r\}=\frac{1}{200} \sum_{i=0}^{200} \frac{1}{N_{s t e p}} \sum_{t=0}^{N_{s t e p}} r_{t}$ i.e. one episode consists of duration $N_{\text {step }}$ ). It can be observed that DQN converge to a optimal value function $Q^{*}(s, a)$ over 2500 training episodes. The increase in average reward over training episodes in all the observations demonstrate that DQN can successfully learn the optimal trajectory for UAV along with BS beam directions.

Red colored path along the grid in Fig. 4 shows the UAV trajectory of proposed DQN-agent based joint path planning and beam tracking method for a random test episode starting 


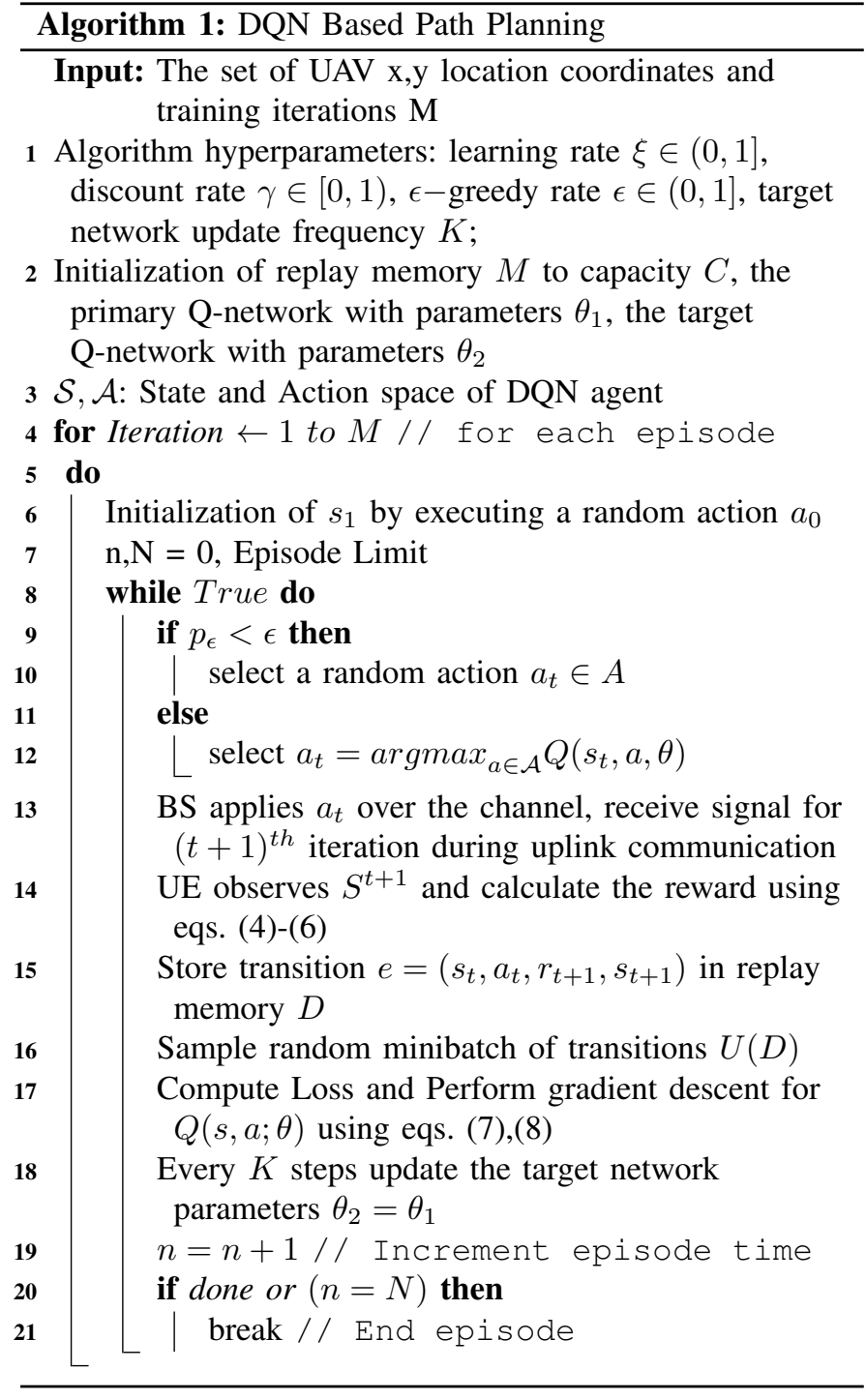

from source $\left(\mathrm{UE}_{s}=(450,450)\right)$ and reaching towards destination $\left(\mathrm{UE}_{d}=(-500,-500)\right)$. Similarly, black colored path along the grid represents learnt shortest path planning UAV trajectory using a different RL BS agent under same simulation conditions. The BS for this scenario is located at $(0,0)$ as shown in Fig. 4. We consider a multi-path communication model scenario with 3 reflection points at $\mathrm{rp}_{1}=(0,150), \mathrm{rp}_{2}=(250,50)$ and $\mathrm{rp}_{3}=(-200,-150)$. The DQN-agent used for the proposed path is trained over 2500 episodes. For the shortest path planning method, we retrained the same DQN-agent by computing rewards without rate conditions. A heuristic exhaustive beam search method is separately performed later at every location along the learnt path, computing the best possible data rate measurements.

We observe that DQN approach follows a different path compared to shortest path in reaching the same target location. Despite different optimization criteria, both the methods are able to reach the destination in an equal number of steps. Thus, the DQN approach can offer as a generic framework to learn
TABLE I

SIMULATION PARAMETERS

\begin{tabular}{|c|c|}
\hline Parameters & Value \\
\hline mmWave Channel & UMa \\
UMa-LoS Pathloss coefficients & $\{\alpha: 2.8, \beta: 11.4, \gamma: 2.3, \sigma: 4.1\}$ \\
UMa-NLoS Pathloss coefficients & $\{\alpha: 3.3, \beta: 17.6, \gamma: 2.0, \sigma: 9.9\}$ \\
mmWave freq & $30 \mathrm{GHz}$ \\
carrier spacing freq $d f$ & $60 \mathrm{kHz}$ \\
Num of subspace carriers NFFT & 1200 \\
antenna element spacing $d$ & 0.5 \\
Transmit power $P_{\mathrm{tx}}$ & $30 \mathrm{dBm}$ \\
Transmit antenna elements $N_{t}$ & 8 \\
Receiving antenna elements $N_{r}$ & 8 \\
Noise Level $N_{0}$ & $-174 \mathrm{dBm}$ \\
BS location & {$[0,0,0]$} \\
coverage xloc $\mathbb{U}_{\text {xloc }}$ & {$[-500,500,50] \mathrm{m}$} \\
coverage yloc $\mathbb{U}_{\text {yloc }}$ & {$[0] \mathrm{m}$} \\
coverage zloc $\mathbb{U}_{\text {zloc }}$ & $800,50] \mathrm{m}$ \\
Cardinality of Beamset $M=|\mathcal{B}|$ & \\
\hline
\end{tabular}

TABLE II

DQN HYPER-PARAMETERS

\begin{tabular}{|c|c|}
\hline Hyperparameters & Value \\
\hline Learning rate $\lambda_{\text {Adam }}$ & $5 e^{-4}$ \\
$\epsilon-$ start & 1.0 \\
$\epsilon$-end & 0.01 \\
$\epsilon-$ decay & 0.9983 \\
Soft-Update rate $\tau$ & 0.001 \\
Target Q-Network Update Frequency $K$ & 10 \\
Minbatch Size $U(D)$ & 64 \\
Replay Memory Size $|D|$ & $10^{5}$ \\
Discount rate $\gamma$ & 0.999 \\
\hline
\end{tabular}

the optimal trajectories for UAV under different constraint conditions. Also, the computed data rate measurements (in Gbps) are labelled in a bounding box (following color coding) at every location along the path, for both the methods. Rate measurements along the red path are learnt directly from the joint path-planning and beam tracking DQN approach while the measurements along black path are computed using heuristic exhaustive beam search method following multipath channel conditions. We observe that red labelled learnt data rate values are comparable to that of heuristic grey labelled values, justfying the beam learning under multi-path conditions. The learnt beam directions at each location along the path, prevents frequent beam scanning at BS, reducing its communication overhead to a great extent. This can be very energy efficient especially when the BS commands multiple UAVs at the same time. BS considers a prior computational overhead by learning optimized UAV trajectories within its coverage area, based on its constraints and prevent frequent beam sweeping overhead all the time.

However, the accuracy of the learnt data rate values still needs to be analyzed. This can be better understood by considering learnt and heuristic data rate values along red and blue line respectively, starting from same random source location as shown in Fig. 5. We observe that increase in cumulative rates along the blue curve is higher compared to that of cumulative rates in red plot. This indicates that the learnt rate values are comparable to heuristic approach but the 
average learnt rate measurement for a location is slightly lower than its average exhautive rate measurement along the similar joint path planning and beam tracking path. The difference in measurements is due to joint optimization of target distance and receiver beam direction at $\mathrm{BS}$, during path planning. This justifies that the proposed approach jointly optimize the trajectory as well as achieve mmWave data rates comparable to that of exhaustive method, using beam tracking.

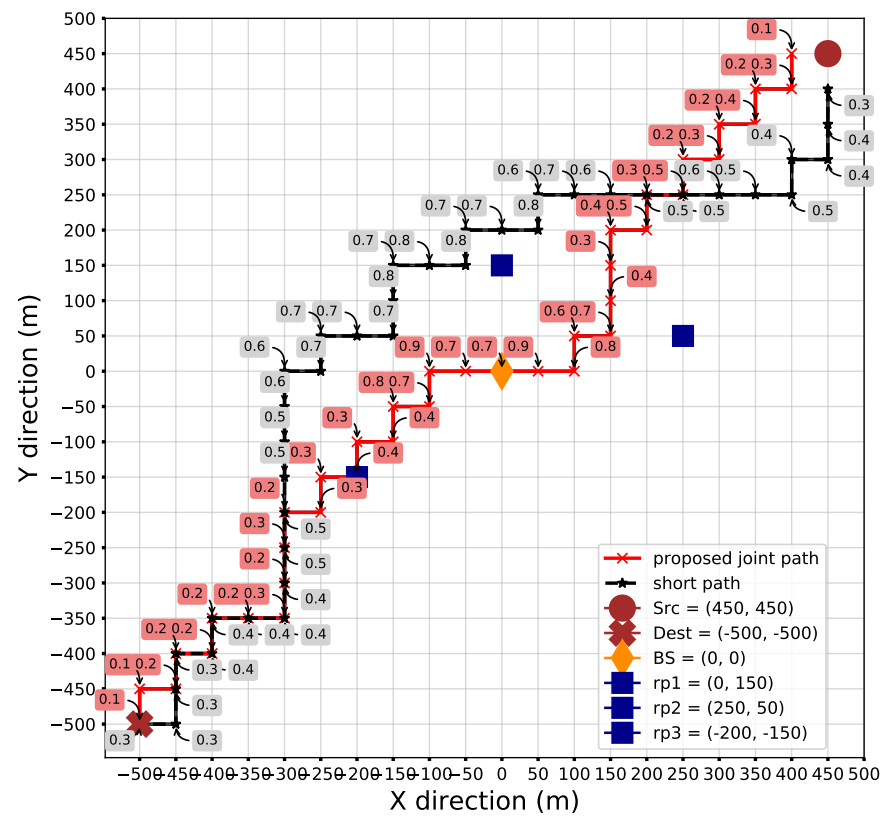

Fig. 4. Joint path planning and beam tracking trajectory as well as shortest path planning trajectory for a random DQN test episode of source $(450,450)$ and destination $(-500,-500)$

\section{CONCLUSion AND Future WORK}

We developed a deep RL based uplink trajectory optimization framework for cellular-enabled UAVs within the coverage area of BS. We confined our UE constraints to target distance and data rate measurements while designing the generic framework using DQN algorithm. We initially shown the converge performance of DQN algorithm and then analyzed the results of joint path planning and beam tracking based trajectory against learnt shortest path planning towards destination. We also compare the performance of learnt rate measurements against the heuristic exhaustive beam search method. Thus, the proposed approach provides a generic framework for jointly optimizing multiple practical constraints to the path planning problem via BS. Having demonstrated some promising results, we would like to consider other constraints such as energy, fading and other complex terrestial channel conditions in a multi-UAV scenario around BS, as extension to this research in future.

\section{ACKNOWLEDGEMENT}

The research leading to these results has received funding from European-Union project Primo-5G, Academy of Finland projects 6Genesis Flagship (Grant No. 318927), IIoT

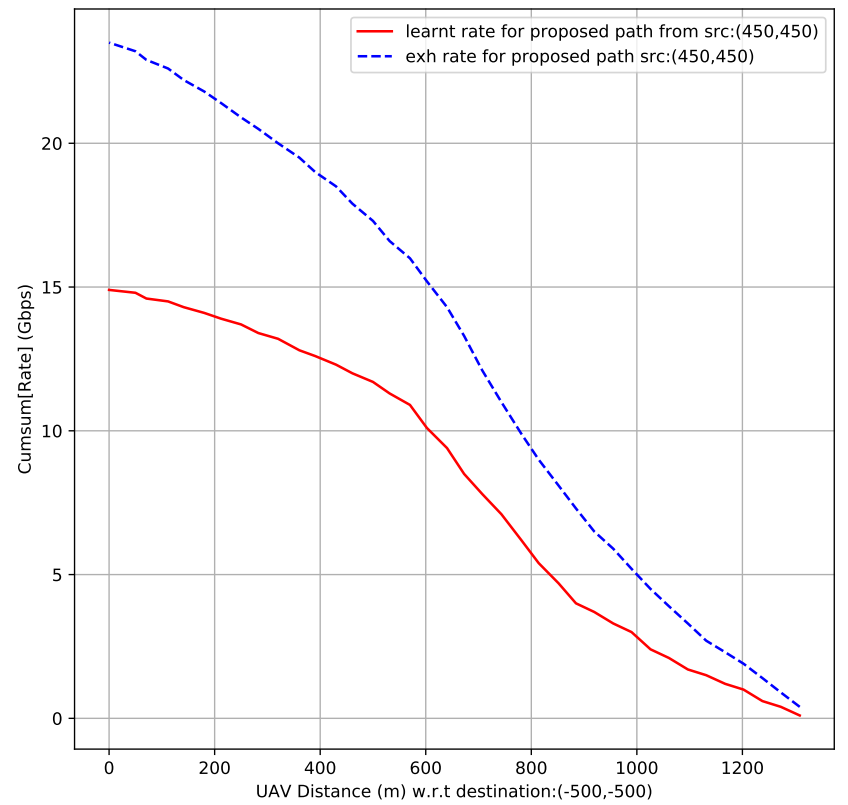

Fig. 5. Cumulative exhaustive and learnt rates along joint path planning and beam tracking trajectory across UAV distance with respect to its destination location

CONnectivity for mechanICAL systems (ICONICAL) and Positioning-aided Reliably-connected Industrial Systems with Mobile mmWave Access (PRISMA).

\section{REFERENCES}

[1] S. D. Muruganathan, X. Lin, H.-L. Maattanen, Z. Zou, W. A. Hapsari, and S. Yasukawa, "An overview of 3GPP release-15 study on enhanced LTE support for connected drones," arXiv preprint arXiv:1805.00826, 2018.

[2] L. Zhang, H. Zhao, S. Hou, Z. Zhao, H. Xu, X. Wu, Q. Wu, and R. Zhang, "A Survey on 5G Millimeter Wave Communications for UAVAssisted Wireless Networks," IEEE Access, vol. 7, pp. 117 460-117 504, 2019.

[3] L. Gupta, R. Jain, and G. Vaszkun, "Survey of important issues in uav communication networks," IEEE Communications Surveys \& Tutorials, vol. 18, no. 2, pp. 1123-1152, 2015.

[4] S. Hayat, E. Yanmaz, and R. Muzaffar, "Survey on Unmanned Aerial Vehicle Networks for Civil Applications: A Communications Viewpoint," IEEE Communications Surveys \& Tutorials, vol. 18, no. 4, pp. 26242661, 2016.

[5] "What the Amazon Effect Means for the Shipping Industry," Jun 2019 [Online]. Available: https://www.shipware.com/what-the-amazon-effectmeans-for-the-shipping-industry/

[6] R. W. Heath, N. Gonzalez-Prelcic, S. Rangan, W. Roh, and A. M. Sayeed, "An overview of signal processing techniques for millimeter wave mimo systems," IEEE journal of selected topics in signal processing, vol. 10, no. 3, pp. 436-453, 2016.

[7] P. Wang, Y. Li, L. Song, and B. Vucetic, "Multi-gigabit millimeter wave wireless communications for 5g: From fixed access to cellular networks," IEEE Communications Magazine, vol. 53, no. 1, pp. 168-178, 2015.

[8] G. Yue, Z. Wang, L. Chen, L. Cheng, J. Tang, X. Zou, Y. Zeng, and L. Li, "Demonstration of $60 \mathrm{ghz}$ millimeter-wave short-range wireless communication system at 3.5 gbps over $5 \mathrm{~m}$ range," Science China Information Sciences, vol. 60, no. 8, p. 080306, 2017.

[9] D. Nandi and A. Maitra, "Study of rain attenuation effects for $5 \mathrm{G} \mathrm{mm-}$ wave cellular communication in tropical location," IET Microwaves, Antennas \& Propagation, vol. 12, no. 9, pp. 1504-1507, 2018.

[10] G. Wild, J. Murray, and G. Baxter, "Exploring civil drone accidents and incidents to help prevent potential air disasters," Aerospace, vol. 3, no. 3, p. 22,2016 
[11] B. Pearre and T. X. Brown, "Model-free trajectory optimization for wireless data ferries among multiple sources," in 2010 IEEE Globecom Workshops. IEEE, 2010, pp. 1793-1798.

[12] S. Zhang, H. Zhang, B. Di, and L. Song, "Joint Trajectory and Power Optimization for UAV Sensing Over Cellular Networks," IEEE Communications Letters, vol. 22, no. 11, pp. 2382-2385, 2018.

[13] Q. Wu, Y. Zeng, and R. Zhang, "Joint Trajectory and Communication Design for Multi-UAV Enabled Wireless Networks," IEEE Transactions on Wireless Communications, vol. 17, no. 3, pp. 2109-2121, 2018.

[14] U. Challita, W. Saad, and C. Bettstetter, "Deep reinforcement learning for interference-aware path planning of cellular-connected UAVs," in 2018 IEEE International Conference on Communications (ICC). IEEE, 2018, pp. 1-7.

[15] V. Va, T. Shimizu, G. Bansal, and R. W. Heath, "Online Learning for Position-Aided Millimeter Wave Beam Training," IEEE Access, vol. 7, pp. 30507-30 526, 2019.

[16] S. Amuru, "Beam Learning-Using Machine Learning for Finding Beam Directions," arXiv preprint arXiv:1906.04368, 2019.

[17] A. Sawant, "UAV Battery Market 2018-2023: Global Size, Share, Trends, Leading Players and Regional Analysis By UAV and Battery Type." [Online]. Available: https://www.reuters.com/brandfeatures/venture-capital/article?id=48656

[18] J. Campos, "Understanding the 5G NR Physical Layer," Keysight Technologies, 2017.

[19] S. Sun, T. S. Rappaport, S. Rangan, T. A. Thomas, A. Ghosh, I. Z. Kovacs, I. Rodriguez, O. Koymen, A. Partyka, and J. Jarvelainen, "Propagation path loss models for 5g urban micro-and macro-cellular scenarios," in 2016 IEEE 83rd Vehicular Technology Conference (VTC Spring). IEEE, 2016, pp. 1-6.

[20] R. S. Sutton and A. G. Barto, Reinforcement learning: An introduction. MIT press, 2018.

[21] V. Mnih, K. Kavukcuoglu, D. Silver, A. A. Rusu, J. Veness, M. G. Bellemare, A. Graves, M. Riedmiller, A. K. Fidjeland, G. Ostrovski et al., "Human-level control through deep reinforcement learning," Nature, vol. 518, no. 7540, p. 529, 2015.

[22] G. Brockman, V. Cheung, L. Pettersson, J. Schneider, J. Schulman, J. Tang, and W. Zaremba, "Openai gym," arXiv preprint arXiv:1606.01540, 2016.

[23] K. He, X. Zhang, S. Ren, and J. Sun, "Delving deep into rectifiers: Surpassing human-level performance on imagenet classification," in Proceedings of the IEEE international conference on computer vision, 2015, pp. 1026-1034.

[24] C. M. Bishop, Pattern Recognition and Machine Learning. Springer, 2006.

[25] D. P. Kingma and J. Ba, "Adam: A method for stochastic optimization," arXiv preprint arXiv:1412.6980, 2014.

[26] J. Saloranta, G. Destino, and H. Wymeersch, "Comparison of different beamtraining strategies from a rate-positioning trade-off perspective," in 2017 European Conference on Networks and Communications (EuCNC). IEEE, 2017, pp. 1-5. 